\title{
Colorectal cancer: a multifaceted disease
}

We are pleased to present this focused issue on "Molecular Markers in Colorectal Cancer". Advances in molecular profiling and bioinformatics have opened a frontier of exponentially increasing new knowledge that has accelerated the promise of precision oncology. The articles in this issue of Chinese Clinical Oncology highlight the genetic heterogeneity of colorectal cancer and biomarkers that predict prognosis and response to therapy. We thank the authors of this issue whose contributions have transformed our understanding of colorectal cancer biology and led to improvements in patient survival.

\section{Acknowledgments}

None.

\section{Footnote}

Conflicts of Interest: The authors have no conflicts of interest to declare.

Ethical Statement: The authors are accountable for all aspects of the work in ensuring that questions related to the accuracy or integrity of any part of the work are appropriately investigated and resolved.

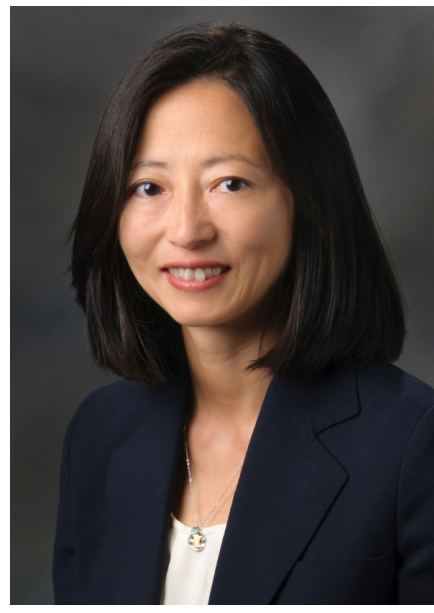

Yun Shin Chun

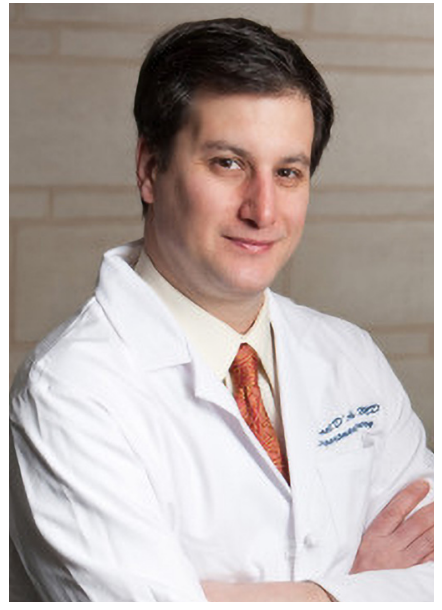

Michael D'Angelica

Yun Shin Chun

The University of Texas MD Anderson Cancer Center, Houston, TX, USA;

(Email:yschun@mdanderson.org)

Michael D'Angelica

Memorial Sloan Kettering Cancer Center, New York, NY, USA.

(Email:dangelim@mskcc.org)

doi: $10.21037 /$ cco.2019.09.02

Cite this article as: Chun YS, D'Angelica M. Colorectal cancer: a multifaceted disease. Chin Clin Oncol 2019;8(5):45. doi: 10.21037/cco.2019.09.02 